\title{
Engineering of Long-Term Stable Transparent Nanoemulsion Using High-Gravity Rotating Packed Bed for Oral Drug Delivery
}

This article was published in the following Dove Press journal: International Journal of Nanomedicine

\author{
Hao-Ran $\mathrm{Wu}^{\prime}$ \\ Chuan-Qi Wang (ID) \\ Jie-Xin Wang ${ }^{1,2}$ \\ Jian-Feng Chen ${ }^{1,2}$ \\ Yuan Le ${ }^{1,2}$
}

'State Key Laboratory of OrganicInorganic Composites, Beijing University of Chemical Technology, Beijing I00029, People's Republic of China; ${ }^{2}$ Research Center of the Ministry of Education for High Gravity Engineering and Technology, Beijing University of Chemical Technology, Beijing 100029, People's Republic of China
Correspondence: Yuan Le State Key Laboratory of OrganicInorganic Composites, Beijing University of Chemical Technology, Beijing, Chaoyang District 100029, People's Republic of China

Email leyuan@mail.buct.edu.cn
Background: Oil-in-water drug nanoemulsion forms drug delivery systems with high oral bioavailability. The conventional fabrication methods of nanoemulsion are low energy emulsification methods and high energy emulsification methods. However, both two methods are not ideal for industrial production. The problem of low energy emulsification methods is the high dosage of surfactant and co-surfactant which has potential biosecurity issues. What is more, high energy emulsification methods have some disadvantages, like the destruction of drug components, the price of equipment and the difficulties of industrial production. Hence, there have been a few commercial drug nanoemulsions so far.

Methods: In this work, we reported a novel method for the fabrication of stable and transparent drug nanoemulsion which contains hydrophilic drug rosuvastatin (ROS) calcium or hydrophobic drug silybinin (SYN) by using high-gravity rotating packed bed (RPB). The drug nanoemulsion was systematically characterized by droplet size, size distribution, stability and in vitro drug release as well as Caco-2 cells permeability.

Results: Compared with the self-emulsification method (SE), high-gravity technology could reduce $75 \%$ amount of mixed surfactants. The as-prepared nanoemulsion exhibited a very narrow droplet size distribution with a size of $13.53 \pm 0.53 \mathrm{~nm}$ and a polydispersity index of $0.073 \pm 0.018$. Meanwhile, the drug nanoemulsion was physicochemically stable at $25^{\circ} \mathrm{C}$ and $4^{\circ} \mathrm{C}$ for one-year storage. Furthermore, both ROS and SYN nanoemulsion displayed higher cell permeability and in vitro dissolution than that of commercial formulations.

Conclusion: These results demonstrate that RPB can be a potential device to facilitate the industrial production of drug nanoemulsion.

Keywords: nanoemulsion, high-gravity rotating packed bed, Rosuvastatin calcium, Silybinin, stability, in vitro dissolution, Caco-2 permeability

\section{Introduction}

Oral route is the most common and preferred route of administration due to its painless convenience and cost-effectiveness. Oral bioavailability of drugs is strongly influenced by their formulations. ${ }^{1}$ Nanoemulsions are considered as an ideal alternative for the oral administration of drugs because they exhibit various advantages such as high solubilization capacity for both hydrophilic and hydrophobic drugs due to the presence of emulsifier-based interface between oil and water, 2,3 ability to improve lymphatic absorption henceforth avoiding the first-pass metabolism and enhancing bioavailability. ${ }^{4,5}$ Some of the drug nanoemulsions, ie cyclosporine $\left(\right.$ Neoral $^{\circledR}$, Gengraf $^{\circledR}$ ), saquinavir (Fortovase ${ }^{\circledR}$ ), and ritonavir $\left(\right.$ Norvir $\left.^{\circledR}\right)$ have been approved by the FDA for clinical uses. ${ }^{6}$ Fabrication 
methods for nanoemulsion can be classified as low energy emulsification methods and high energy emulsification methods. ${ }^{7}$ Low energy methods involve spontaneous emulsification (SE) and phase inversion. ${ }^{8} \mathrm{Up}$ to now, the commercial formulation of nanoemulsion was made by using these methods. ${ }^{9,10}$ A key character of these methods is the utilization of energy stored in the system to produce ultra-fine droplets. Low energy methods are sometimes limited by oil type and emulsifiers that can be used, and also required a large amount of surfactants for stabilization of droplets, which can cause biomembrane fluidization. ${ }^{11}$ High energy emulsification is widely used in industrial operations because of the flexible control of emulsion droplet size distribution and the ability to produce fine emulsions from a wide variety of materials. ${ }^{12}$ High-energy emulsification techniques include high-pressure homogenization, microfluidizer and ultrasonication. ${ }^{11}$ External energy is given in the form of shear, ultrasonic waves and pressure, which breaks the droplets in nanosize range. ${ }^{13}$ However, extreme heat generated during these processes may cause decomposition of active pharmaceutical ingredients or surfactant molecules. ${ }^{8}$ On the other hand, equipment of these techniques are expensive and difficult to mass-produce and all these methods have not yet been commercialized in the drug delivery system. ${ }^{14-16}$ Hence, developing efficient and largescale preparation methods is still one of the most important tasks for the production of nanoemulsion.

The preparation of nanoemulsions is to mix oil and aqueous phases in the presence of an emulsifier. When the two immiscible phases meet in the reactor, the oil phase diffuses rapidly into the aqueous phase; therefore, a tight control of the mixing process is very important to the preparation of nanoemulsion, because that homogeneous nanodroplets of oil calls for uniformity of spatial distribution of oil droplets in the aqueous phase in molecular scale. Rotating packed bed (RPB), also known as Higee (high gravity), has been used as an effective process intensification technology, which can generate an acceleration of 1-3 orders of magnitude large than gravitational acceleration on the earth. The fluids going through the packing of RPB are spread or split into very fine droplets, threads, and thin films by the strong shear, resulting in a significant intensification of micromixing and mass transfer between the fluid elements. ${ }^{17,18}$ Thus, RPB benefits the form of uniform concentration distribution of fluids. RPB has been proved to be an ideal device for the preparation of nanoparticles and successfully applied in the pharmaceutical industry. ${ }^{19,20}$
To our best knowledge, the preparation of nanoemulsion using high-gravity technology has not been reported until now. The aim of this work was to investigate the feasibility of the preparation of long-term stable nanoemulsion with less surfactant dosage using high-gravity technique. Rosuvastatin calcium (ROS) and Silybinin (SYN) were selected as two model drugs. ROS, a biopharmaceutics classification system (BCS) class III drug, exhibits good water solubility and poor permeability. SYN, a BCSII drug, displays poor water solubility and good permeability. Both drugs show a first-pass metabolism. ${ }^{21,22}$ Droplet size, size distribution, long-term stability were characterized and compared with that prepared by SE method. In vitro dissolution and Caco- 2 cell permeability were performed and compared with the commercial formulation.

\section{Materials and Methods Materials}

ROS and SYN were purchased from Beijing Zhongshuo Pharmaceutical Technology Development Co., Ltd. (Beijing, China). Glyceryl triacetate, opropyl myristate, Olive Oil, EL40 was purchased from Macklin. Soybean oil, corn oil was purchased from Aladdin. RH-40, tween-20 was purchased from Shanghai Yuanye Biotechnology Co., Ltd. (Shanghai, China). Anhydrous ethanol, 1.2-propanediol, n-butanol, 1.4-butanediol, PEG-200 was purchased from Beijing Chemical Works (Beijing, China). ROS commercial tablets purchased from AstraZeneca. SYN commercial capsules purchased from Tianjin Tasly Sants Pharmaceutical Co., Ltd. Pancreatin, penicillin-streptomycin and fetal bovine serum were purchased from Gibco. Dimethyl sulfoxide (DMSO) was purchased from Amresco. Nonessential amino acid was purchased from Macgene Technology (Beijing) Co., Ltd. Caco-2 cells were provided by Peking University Health Science Center.

\section{Equilibrium Solubility Studies}

Oil phase components for the development of nanoemulsion were selected on the basis of equilibrium solubility studies. An excess amount of drug (ROS or SYN) was mixed with the oil phase and kept on a water bath shaker at $37^{\circ} \mathrm{C} \pm 2^{\circ} \mathrm{C}$ for $24 \mathrm{~h}$. Samples were centrifuged at $10,000 \mathrm{rpm}$ for $10 \mathrm{~min}$. The supernatant was analyzed using a validated high-pressure liquid chromatography (HPLC) method. 


\section{Preparation of Nanoemulsion}

Based on our pre-test (pseudo-ternary phase diagram) and determination of drug solubility, we formulated the blank nanoemulsion by glycerol triacetate/RH-40/1, 2-propanediol/aqueous phase $=8.75: 2.5: 1.25: 87.5(\mathrm{w} / \mathrm{w} / \mathrm{w} / \mathrm{w})$. The saturated drug (ROS or SYN) oil solution was first configured and then the excess drug solids were removed by centrifugation at $10,000 \mathrm{rpm}$ for $5 \mathrm{~min}$. After that, the surfactant and co-surfactant were slowly added to the oil phase and vortexed for a period of time (solution A). In this case, solution A and purified water (solution B) were pumped into a high gravity rotating packed bed at a feed ratio of $1: 10(\mathrm{w} / \mathrm{w})$ at a room temperature of $25^{\circ} \mathrm{C}$. It was cycled at $1000 \mathrm{rpm}$ for $10 \mathrm{~min}$. The resulting clear formulation was a drug nanoemulsion. For comparison, we chose a conventional mixing device to prepare another group of nanoemulsions. Solution B was quickly added to solution $\mathrm{A}$ at $25^{\circ} \mathrm{C}$ and stirred at $1000 \mathrm{rpm}$ for $1 \mathrm{~h}$. Then, the collected nanoemulsion is transferred to a container for storage and used for subsequent characterization and experimentation. Each sample was prepared and measured three times separately.

\section{Characterization}

\section{Droplet Size and Polydispersity Index}

The droplet size and PDI value of the different drug nanoemulsion were measured by using ZNANO-ZS90 nanoparticle analyzer (Malvern, UK) at room temperature $\left(25^{\circ} \mathrm{C} \pm\right.$ $0.5^{\circ} \mathrm{C}$ ) which was used dynamic light scattering technique and scattered at an angle of $173^{\circ}$. For the measurement, the samples were diluted with deionized water (1:200) and injected into the sample cell. Intensity distribution was used for the measurement of mean average droplet size.

\section{Tem}

The morphology of the nanoemulsions were examined by an Energy-Filtering Transmission electron microscopy (TEM) (Hitachi HT7700, Japan) with an $80 \mathrm{kV}$ accelerating voltage. The nanoemulsions were negatively stained by $2 \%$ phosphotungstic acid (PTA) and placed on carboncoated 400 mesh copper grids followed by drying at room temperature before measurements.

\section{High-Performance Liquid Chromatography (HPLC)}

The drug content of ROS or SYN was quantified by HPLC (Waters 2695, USA). After being filtered, the samples were dissolved and diluted by mobile phase solvent—sulfuric acid solution (0.03\%)-acetonitrile (46:54). Ascentis C18 column was equipped in the mobile phase. The optimal detection wavelength of ROS was $242 \mathrm{~nm}$ and SYN was $288 \mathrm{~nm}$, and the column temperature was $40^{\circ} \mathrm{C}$. The flow rate was $1 \mathrm{~mL} /$ min and the injection volume was $10 \mu \mathrm{L}$. Serially diluted samples of ROS or SYN dissolved in the mobile phase solvent $(0.5,0.8,1,2,5,10,20,50,80$ and $100 \mu \mathrm{g} / \mathrm{mL})$ and were prepared as standards for the HPLC analysis. The standard curves of both drugs were shown in Figure S1.

\section{Stability Study Accelerate Experiments}

About $15 \mathrm{~mL}$ of the prepared drug nanoemulsion was stored in a $20 \mathrm{~mL}$ clean clear glass bottle and tested for stability within 2 weeks at high temperature $\left(4^{\circ} \mathrm{C}\right.$ and $\left.60^{\circ} \mathrm{C}\right) .{ }^{23,32}$ Among them, samples were taken on the 1st, 5th, 7th, 10th, and 14th day, respectively. Centrifugal stability was tested by a high-speed centrifuge and the nanoemulsion was centrifuged at 10,000 rpm for $30 \mathrm{~min}$. The appearance, the average droplet size, and the polydispersity index (PDI) and the drug content were then observed to evaluate the physical stability of the drug nanoemulsion. The amount of drug was determined by HPLC and the droplet size and PDI were determined by a NANO-ZS90 nanoparticle analyzer.

\section{Long-Term Stability}

About $15 \mathrm{~mL}$ of the prepared the drug nanoemulsion was stored in a $20 \mathrm{~mL}$ clean clear glass bottle and tested for a long period stability of 1 year at $4^{\circ} \mathrm{C}$ and $25^{\circ} \mathrm{C} .{ }^{23,32}$ Among them, the samples were taken on the half a month, 1 month, 3 months, 6 months and 1 year, respectively. Centrifugal stability was tested by a high-speed centrifuge and the nanoemulsion was centrifuged at $10,000 \mathrm{rpm}$ for $30 \mathrm{~min}$. The appearance, the average droplet size, and the polydispersity index (PDI) and the drug content were then observed to evaluate the physical stability of the drug nanoemulsion. The amount of drug was determined by HPLC and the droplet size and PDI were determined by a NANO-ZS90 nanoparticle analyzer.

\section{In vitro Dissolution Test}

Dissolution test was performed following the USP Apparatus II (paddle) method by using a dissolution apparatus (D-800LS, Tianjin, China) at $100 \mathrm{rpm}$ and $37.0^{\circ} \mathrm{C} \pm$ $0.5^{\circ} \mathrm{C}$. Phosphate buffer saline (PBS) solution ( $\mathrm{pH} 6.8$ ) with $0.5 \%$ Tween- 80 was employed as the dissolution medium. $1 \mathrm{~mL}$ of SYN nanoemulsion which contains about $3.5 \mathrm{mg}$ SYN drug, and $3.5 \mathrm{mg}$ of commercially available capsule drug were placed in treated dialysis bags, respectively. And 
$5 \mathrm{~mL}$ sample was taken at regular intervals $(0,0.5,1,1.5,2$, $2.5,3,4,5,6,8,12$ and $24 \mathrm{~h}$ ). In the meantime, fresh medium $(5 \mathrm{~mL})$ was added to keep constant volume. The dissolution test of each sample was performed in triplicate.

\section{Transport Study of Drug Nanoemulsion}

For the in vitro transport study of drug nanoemulsion, the colonic adenocarcinoma cell line (Caco-2 cells) obtained from Peking University Health Science Center (Beijing, China) was cultured in Dulbecco's modified eagle medium (DMEM) containing 10\% fetal bovine serum, 1\% nonessential amino acid solution and $1 \%$ penicillin streptomycin at $37^{\circ} \mathrm{C}$ in an atmosphere of $5 \% \mathrm{CO}_{2}$.

MTT assay was conducted to investigate the cytotoxic effects of each drug nanoemulsion's concentrations (1, 5, $10,20,40,60,80,100,200 \mu \mathrm{g} / \mathrm{mL}$ ) on Caco-2 cells. Caco2 cells were harvested and seeded in standard 96-well microplates at a density of $6 \times 103$ cells/well and treated with nanoemulsion solution and the control (PBS) for $2 \mathrm{~h}$. The cells were then incubated with MTT $(20 \mu \mathrm{L}, 5 \%)$ at $37^{\circ} \mathrm{C}$ for $4 \mathrm{~h}$, added $100 \mu \mathrm{L}$ DMSO and incubated for 10 min at $37^{\circ} \mathrm{C}$. Optical density (OD) was observed at $570 \mathrm{~nm}$ using a Microplate Reader. Cell viability was calculated according to: cell viability $(\%)=($ mean OD of experiment/mean OD of control) $* 100 \%$.

Determination of the Permeation Rate Across Caco-2 Cell Monolayers. The procedures for Caco-2 cell monolayers permeation assay followed others' study. ${ }^{24}$ Briefly, for apical to basolateral (A to B) transport study, $0.5 \mathrm{~mL}$ of each experimental solution was added to the apical side, while $1.5 \mathrm{~mL}$ of Hank's solution was added to the basolateral side, and then cultured at $37^{\circ} \mathrm{C}$ in an atmosphere of $5 \% \mathrm{CO}_{2}$. The $0.5 \mathrm{~mL}$ samples from the basolateral side at collected at the predetermined time intervals of $0,30,60$, $90,120 \mathrm{~min}$ and replaced with fresh medium. For basolateral to apical (B to A) transport study, $1.5 \mathrm{~mL}$ of each experimental solution was added to the basolateral side while $0.5 \mathrm{~mL}$ of Hank's solution was added to the apical side, and then cultured at $37^{\circ} \mathrm{C}$ in an atmosphere of $5 \%$ $\mathrm{CO}_{2}$. The $0.2 \mathrm{~mL}$ samples of the apical side were collected at the predetermined time intervals of $0,30,60,90,120$ min, and replaced with fresh medium. ${ }^{25}$ The concentration of drug was determined by HPLC analysis and the cumulative amount of drug permeation was plotted as a function of time. The apparent permeability coefficient $\left(P_{a p p}\right)$ is determined from the linear slope of the plot using the following equation 1: ${ }^{26}$

$$
\mathrm{P}_{\mathrm{app}}=\frac{\mathrm{dQ}}{\mathrm{dt}} \times \frac{1}{\mathrm{AC}_{0}}
$$

where $P_{a p p}$ is the apparent permeability coefficient $(\mathrm{cm} / \mathrm{s})$, $\frac{\mathrm{dQ}}{\mathrm{dt}}$ is the steady-state flux, $\mathrm{A}$ is the surface area of cell membrane $\left(\mathrm{cm}^{2}\right), C_{0}$ is the initial concentration of drug contents in the apical (for A to B transport) or basolateral (for B to A transport) side.

The efflux rate (ER) was calculated according to the following equation $2:^{27}$

$$
\mathrm{ER}=\frac{\mathrm{P}_{\mathrm{app} \mathrm{ba}}}{\mathrm{P}_{\mathrm{app} \mathrm{ab}}}
$$

where $P_{a p p \quad b a}$ is the permeability coefficient of B-to-A transport; $P_{a p p} a b$ is the permeability coefficient of A-toB transport.

\section{Results and Discussion}

Effect of Surfactants on Droplet Size and Size Distribution

In this work, glycerol triacetate was used as the oil phase, which has significant solvency for both $\operatorname{ROS}\left(37^{\circ} \mathrm{C}, 291.65\right.$ $\pm 8.41 \mathrm{mg} / \mathrm{mL})$ and $\mathrm{SYN}\left(37^{\circ} \mathrm{C}, 35.04 \pm 1.64 \mathrm{mg} / \mathrm{mL}\right)$. In the process of selecting the excipients, the screening of the system can be completed by drawing the pseudo-ternary phase diagram or droplet size screening. Among them, surfactant is one of the most influential factors for the formation of nanoemulsion. We compared five common surfactants, Rh-40/Tween-20/Tween-80/El-40/Span-60.The experimental phenomenon shows that the first four emulsifiers can produce a transparent emulsion, but the droplet size has a significant difference under the same dosage of the different surfactants (Table 1). The use of Rh-40 produces the smallest droplet and polydispersity index, its size is much lower than the others. Taylor's equation emphasizes the key role of amphiphilic surfactants in reducing interfacial tension and affects emulsion stability. The effect of different surfactants is not the same, which may be related to the structure and

Table I The Effect of Different Surfactants on Droplet Size of Nanoemulsion

\begin{tabular}{|l|l|l|}
\hline Surfactants & Droplet Size (nm) & PDI \\
\hline Rh-40 & $15.83 \pm 0.18$ & $0.073 \pm 0.006$ \\
Tween-20 & $97.41 \pm 23.73$ & $0.153 \pm 0.034$ \\
Tween-80 & $165.37 \pm 45.62$ & $0.127 \pm 0.042$ \\
El-40 & $428.94 \pm 105.94$ & $0.147 \pm 0.040$ \\
Span-60 & $976.03 \pm 192.46$ & $0.178 \pm 0.053$ \\
\hline
\end{tabular}


hydrophilic-lipophilic balance (HLB) of the surfactant, and determines the type of nanoemulsions that is ultimately obtained.

The type of co-surfactant, the ratio of surfactant to cosurfactant $(\mathrm{Km})$, was also selected by the method described above. We compared five common co-surfactants, anhydrous ethanol/1,2-propanediol/n-butanol/1,4-butanediol/PEG-200 and different values of $\mathrm{Km}, 4: 1 / 2: 1 / 1: 1 / 1: 2 / 1: 4$. We chose glycerol triacetate, RH40 and 1, 2 propanediol, and the value of $\mathrm{Km}$ is $2: 1$ as the formulation of nanoemulsion.

What is more, we did the experiment on the ratio of oil phase to mixed surfactant (1:9 to 9:1) by self-emulsification method (SE) and successfully produced a transparent emulsion in a certain range on the ratio of oil phase to mixed surfactant (1:9 to 7:3). We chose 7:3 as the ratio of oil phase to mixed surfactant because the minimum amount of surfactant and co-surfactant can ensure the impact of excipients used on biological security. As the oil-in-water nanoemulsion, the critical amount of aqueous phase was chosen as the formula and the ratio of aqueous phase to other phases was 10:1. Finally, we formulated the nanoemulsion by glycerol triacetate/RH-40/1, 2-propanediol/aqueous phase $=8.75: 2.5: 1.25: 87.5(\mathrm{w} / \mathrm{w} / \mathrm{w} / \mathrm{w})$. The pseudo-ternary phase diagram of this system provides a reference for preparation of RPB samples (Figure 1).

Based on the clinic dosage of ROS, we investigated the influence of drug loading $(0,25,50,75,100 \mathrm{mg} / \mathrm{mL})$ on the droplet size and polydispersity index (Figure 2). The drug

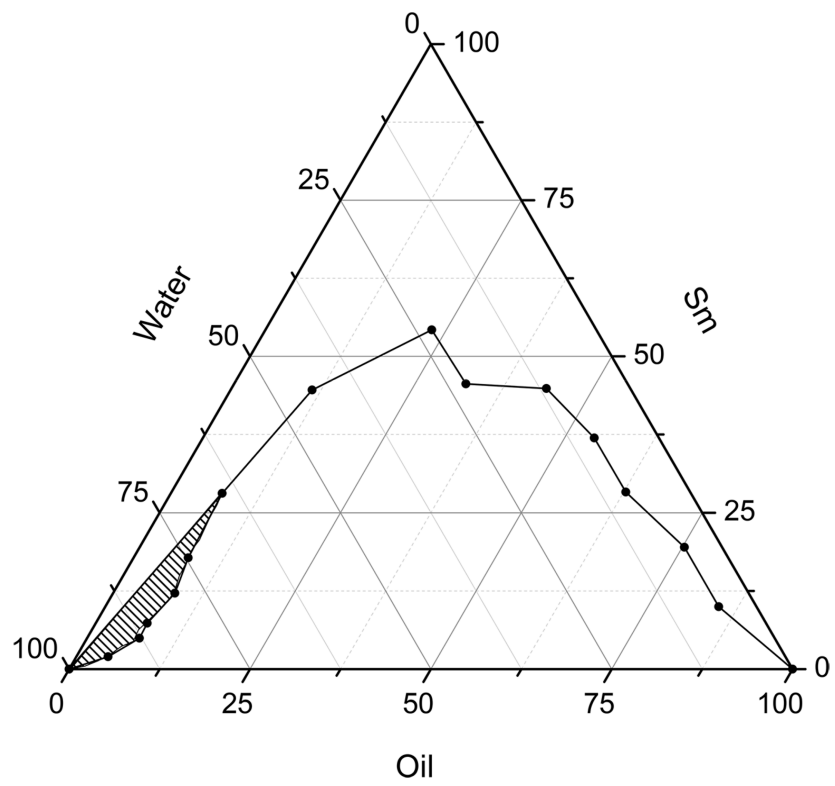

Figure I The pseudo-ternary phase diagram of the selected system. Abbreviation: Sm, Surfactant mixed. loading amount can hardly affect the size of droplets. Because the drug was dissolved in oil phase and existed as a drug molecular form during the mixing process. The drug molecules were protected by emulsion. Nanoemulsion transported the drug molecules into the bloodstream like carriers. We can also know from the TEM image (Figure S2) that the drug loading (SYN) has no influence on the droplet size and the morphology of nanoemulsion.

\section{Preparation of the Nanoemulsion by RPB} Generally speaking, an ideal oral emulsion formulation is supposed to form stable small droplets using the lowest amount of emulsifiers for economic, taste, and safety reasons. Thus, we try to decrease the amount of surfactants with the help of RPB. High-gravity level $\beta$, an important parameter, describes the strength of the high-gravity field, which also represents the intensification degree of the mass transfer and micromixing. ${ }^{28}$ The $\beta$ has a few effects on the droplet size, firstly, the size decreased a little with the $\beta$ increase; then, it kept almost unchanged when the $\beta$ continuously increased (Figure 3). Because the oil droplets are so soft, they are easily broken up into smaller droplets in the high-gravity field. When fluid turbulence in the RPB was intense at high $\beta$, the formation of large droplets was subjected by strong shear forces. Due to the homogeneous space distribution of the droplets in RPB, the size does not significantly change.

Fixing the $\beta$ at 88 , we reduced the amount of surfactants. It is interesting that even if the mixed surfactant is much reduced, the droplet size does not change, and the size distribution is still concentrated. As shown in Figure 4, when the amount of the surfactant is $75 \%, 50 \%, 25 \%$ of the original ratio, small droplets of $14 \mathrm{~nm}$ can still be observed. After 1 month of storage at room temperature, the appearance of

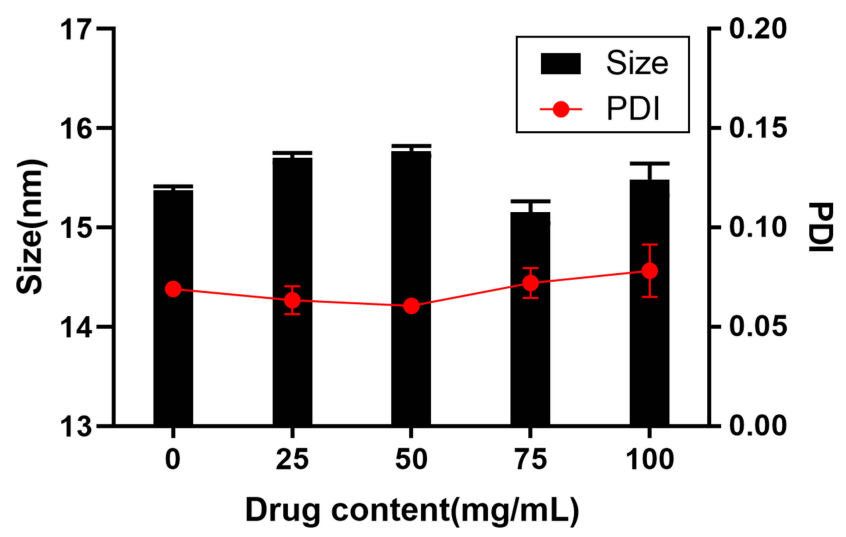

Figure 2 The effect of different drug content on droplet size and PDI of nanoemulsion.

Abbreviation: PDI, polymer dispersity index. 


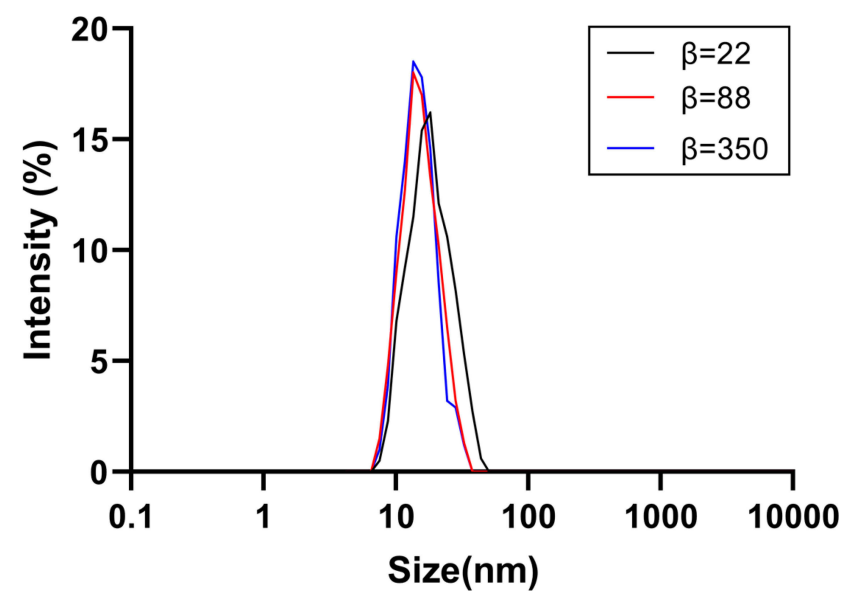

Figure 3 The droplet size distribution at different high-gravity factor $\beta$. Note: $\beta=\frac{\omega \mathrm{r}^{2}}{\mathrm{~g}}$

Abbreviations: $\omega$, rotor angular velocity; $r$, rotor radius; g, gravity acceleration.

these samples remained transparent and showed good fluidity. However, when the amount of surfactant is reduced to $10 \%$ of the SE sample, droplets became larger, and the appearance was turbid after 1 month of storage. The main reason is due to the intensified micro-mixing ability of RPB, in which oil-water interfacial tension is further reduced.
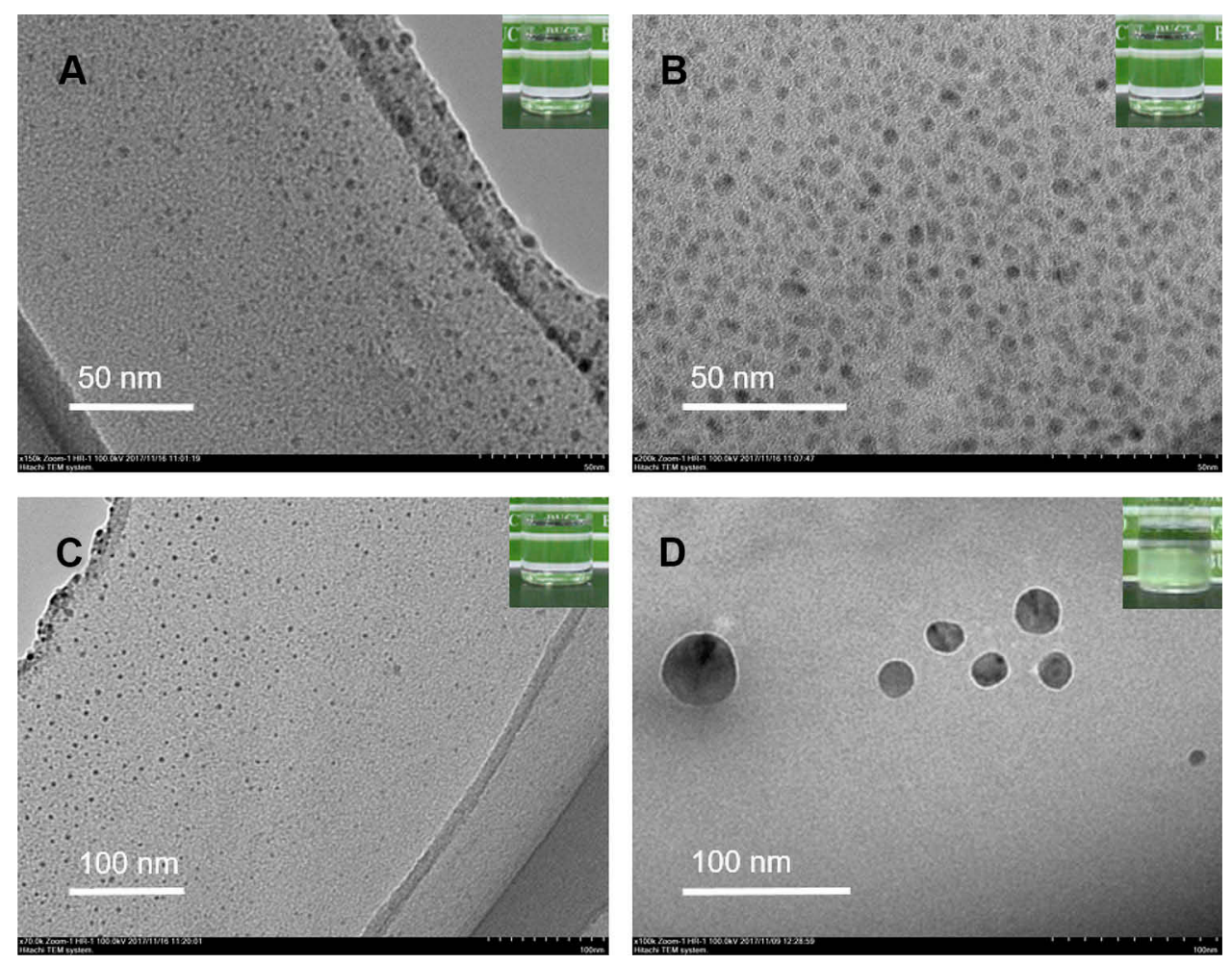

Figure 4 Transmission electron microphotography (TEM) of nanoemulsions prepared by RPB with different surfactant dosage.

Notes: (A) $75 \%$ surfactant dosage of SE samples formulation. (B) $50 \%$ surfactant dosage of SE samples formulation. (C) $25 \%$ surfactant dosage of SE samples formulation. (D) $10 \%$ surfactant dosage of SE samples formulation. Scale bars: A and B $50 \mathrm{~nm}, \mathrm{C}$ and D $100 \mathrm{~nm}$.

Abbreviation: PDI, polymer dispersity index. 


\section{Stability Study}

For practical applications, it is important that emulsionbased delivery systems remain stable during storage and utilization. In this work, both SE nanoemulsion and RPB nanoemulsion contained drug ROS. We first test the effect of temperature on stability. Compared with SE nanoemulsion, samples prepared by RPB displayed more stability at a higher temperature of $60^{\circ} \mathrm{C}$. After storage for 2 weeks at $60^{\circ} \mathrm{C}$, the droplet size of the RPB sample varies only in a small range, only from $12.99 \mathrm{~nm}$ to $15.37 \mathrm{~nm}(\mathrm{p}<0.001)$. However, the SE sample shows strong instability in droplet size, from $14.88 \mathrm{~nm}$ to $593.53 \mathrm{~nm}(\mathrm{p}<0.01)$. The content of both samples decreased, but the drug content of the RPB sample decreased by $21.09 \%(p<0.001)$, which was significantly lower than the $43.67 \%(\mathrm{p}<0.01)$ decrease of the SE sample (Figure 4). The RPB samples stored at $40^{\circ} \mathrm{C}$ exhibited high stability, its droplet size and content remained almost unchanged after 2 weeks of storage. At the same time, although the droplet size of SE samples only changed from $15.15 \mathrm{~nm}$ to $15.73 \mathrm{~nm}(\mathrm{p}<0.001)$, its content still decreased by $12.22 \%(\mathrm{p}<0.01)$ (Figure 5).

And then, 1-year long-term stability test at $25^{\circ} \mathrm{C}$ and $4^{\circ} \mathrm{C}$ represents normal room temperature and frozen storage temperature was tested. The droplet size of the
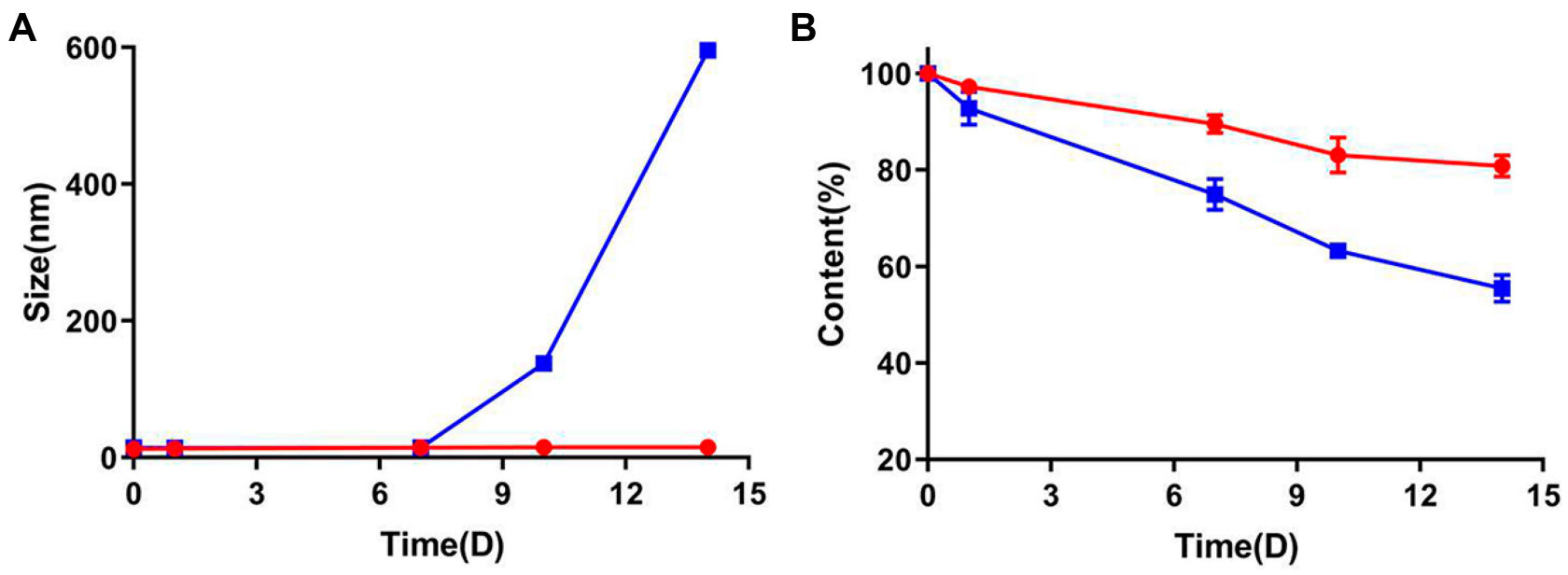

\section{RPB $\rightarrow$ SE $\quad$ Storage temperature: $60^{\circ} \mathrm{C}$}
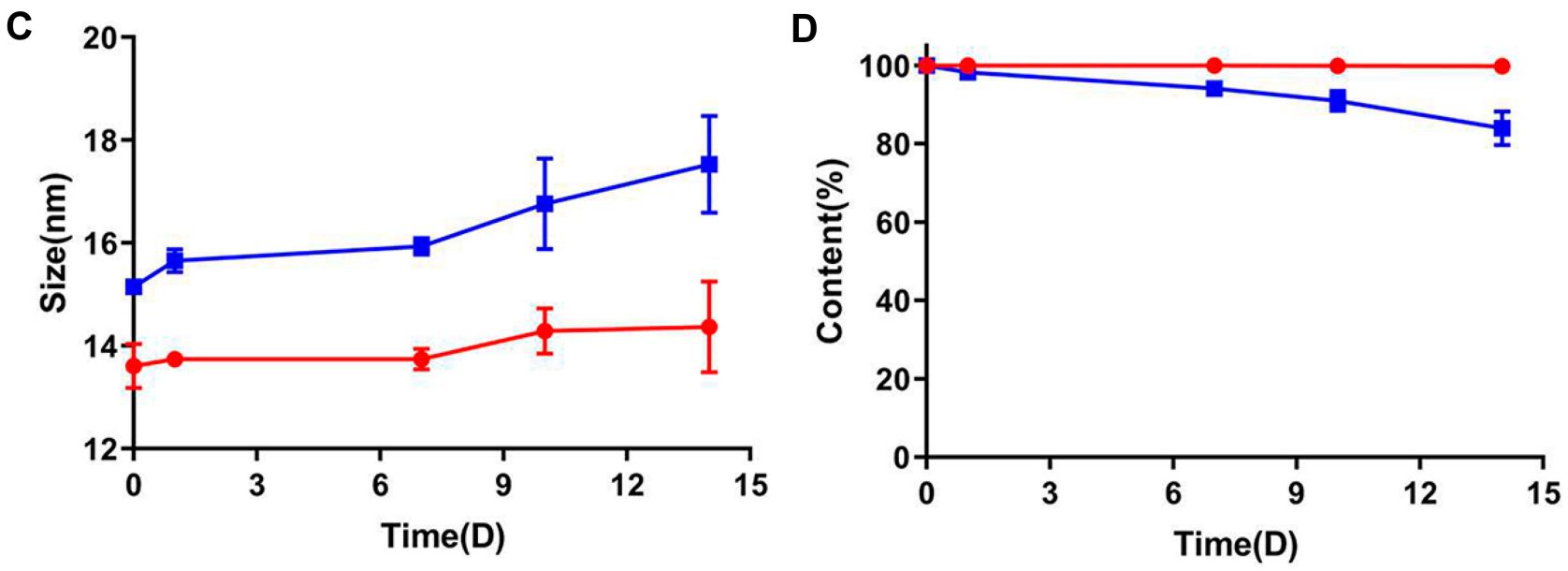

\section{$\rightarrow$ RPB $\rightarrow$ SE $\quad$ Storage temperature: $40^{\circ} \mathrm{C}$}

Figure 5 The sample changes of different nanoemulsion prepared by RPB and SE within 2 weeks.

Notes: (A) Size changes of RPB and SE sample within 2 weeks at $60^{\circ} \mathrm{C}$. (B) Content change of RPB and SE samples within 2 weeks at $40^{\circ} \mathrm{C}$. (C) Size changes of RPB and SE sample within 2 weeks at $40^{\circ} \mathrm{C}$. (D) Content change of RPB and SE samples within 2 weeks at $40^{\circ} \mathrm{C}$.Scale bars: A and B $50 \mathrm{~nm}, \mathrm{C}$ and D $100 \mathrm{~nm}$.

Abbreviations: RPB, high gravity rotating packed bed; SE, self-emulsification method. 
RPB-prepared nanoemulsion did not change significantly both store at $25^{\circ} \mathrm{C}$ and $4^{\circ} \mathrm{C}$ (Figure 6), the average droplet size of the sample is still $15 \mathrm{~nm}(\mathrm{p}<0.001)$. The TEM photos also showed that the PRB sample has almost no change in droplet size and dispersion after 1 year (Figure 7). The drug content of RPB samples decreased by $27 \% \quad(\mathrm{p}<0.01)$ and $17 \% \quad(\mathrm{p}<0.01)$ after storage for 1 year at $25^{\circ} \mathrm{C}$ and $4{ }^{\circ} \mathrm{C}$, the reduction in drug content is largely due to the explanation of the drug's own instability. However, in the case of the nanoemulsion prepared by SE, after a long period of storage, the droplet size gradually increases, after storage for 1 year at $25^{\circ} \mathrm{C}$, the droplet size increases to 259

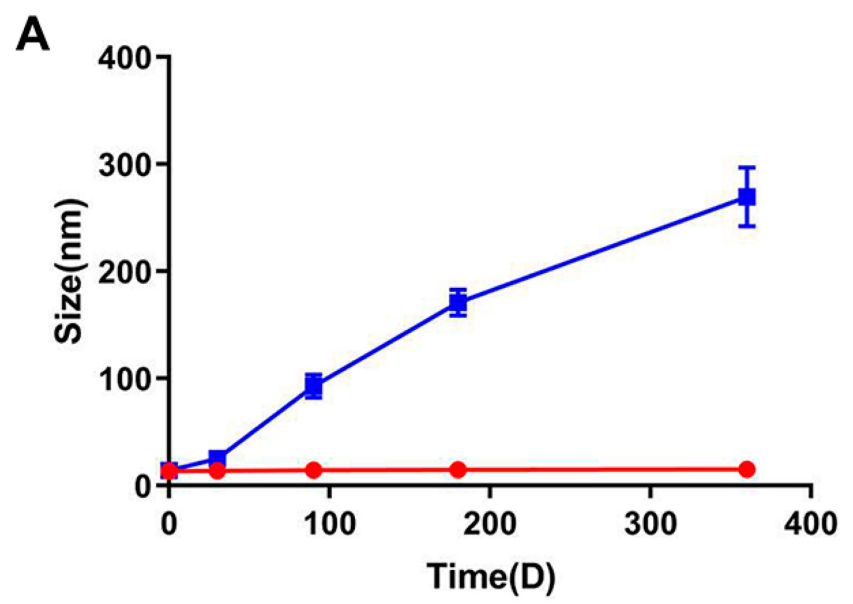

$\mathrm{nm}(\mathrm{p}<0.05)$, and after 1 -yea storage at $4^{\circ} \mathrm{C}$, the droplet size increases to $163 \mathrm{~nm}(\mathrm{p}<0.01)$, the drug content in the SE sample has a further significant decrease relative to the RPB sample, after 1 year of sample storage at $25^{\circ}$ $\mathrm{C}$, the drug content was reduced by $49 \%(\mathrm{p}<0.05)$, and the sample content was reduced by $35 \%(\mathrm{p}<0.05)$ at $4{ }^{\circ} \mathrm{C}$ (Figure 6). Statistically, both RPB samples and SE samples had a significant difference. Intuitively, the appearance of SE samples gradually became cloudy (Figure 7). These phenomena indicate that RPB has great advantages in the preparation of nanoemulsion, the prepared nanoemulsion is more uniform, and the strong micromixing can further reduce the oil-water interfacial
B

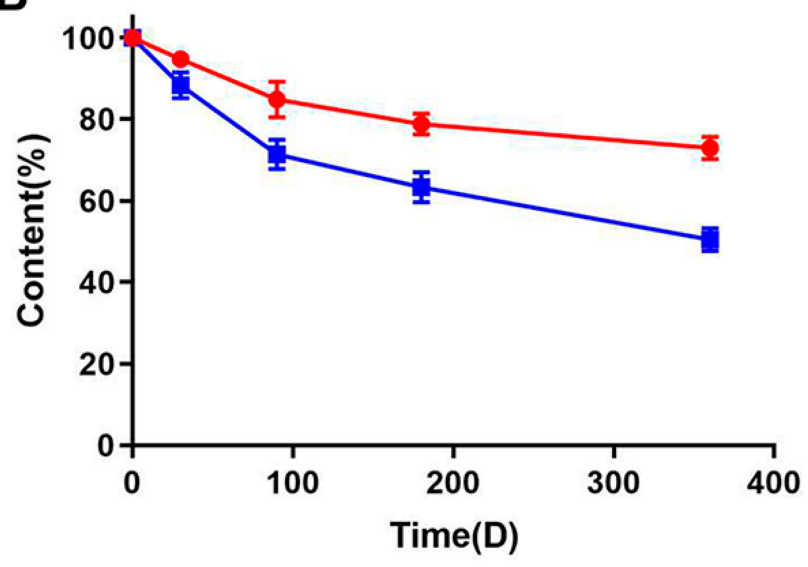

\section{$\mathrm{Rpb} \rightarrow \mathrm{SE} \quad$ Storage temperature $: 25^{\circ} \mathrm{C}$}
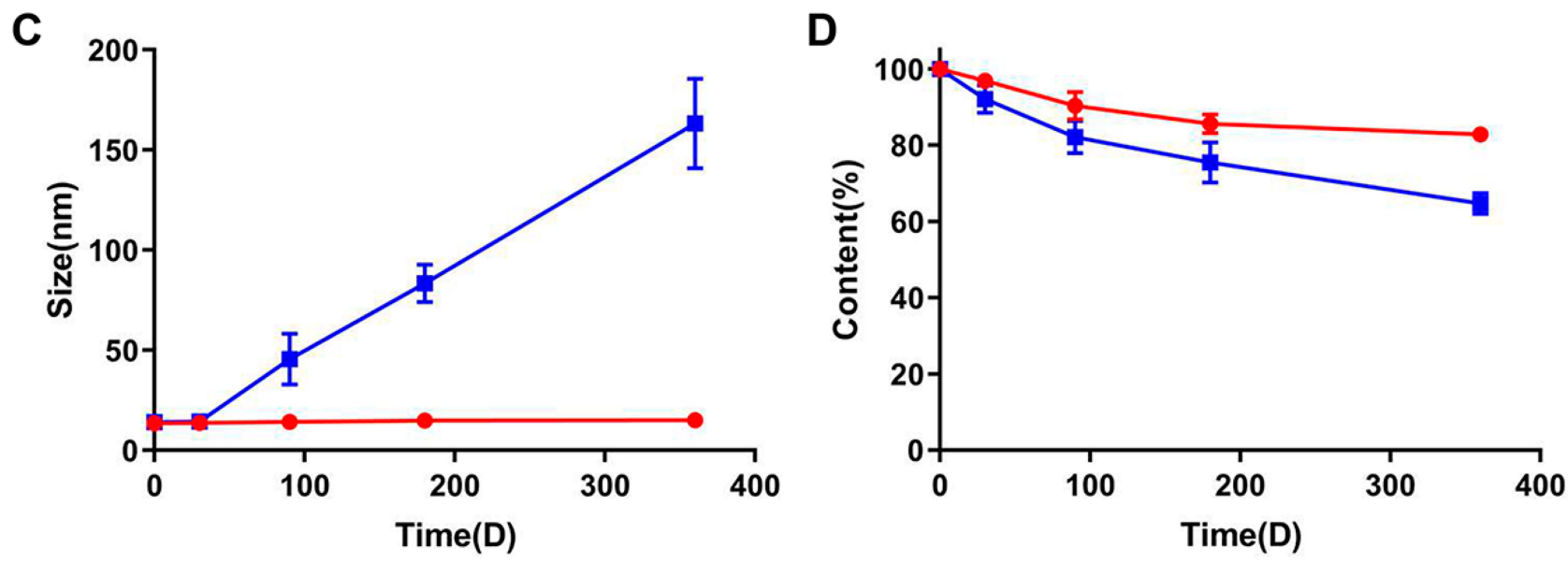

\section{$\rightarrow \mathrm{Rpb} \quad \rightarrow \mathrm{SE} \quad$ Storage temperature: $4^{\circ} \mathrm{C}$}

Figure 6 The samples changes of different nanoemulsion prepared by RPB and SE within I year. A, B at $25^{\circ} \mathrm{C}$ and $\mathrm{C}, \mathrm{D}$ at $4^{\circ} \mathrm{C}$.

Notes: (A) Size changes of RPB and SE sample within 2 weeks at $60^{\circ} \mathrm{C}$. (B) Content change of RPB and SE samples within 2 weeks at $40^{\circ} \mathrm{C}$. (C) Size changes of RPB and SE samples within 2 weeks at $40^{\circ} \mathrm{C}$. (D) Content change of RPB and SE Samples within 2 weeks at $40^{\circ} \mathrm{C}$. Scale bars: A and B $50 \mathrm{~nm}, \mathrm{C}$ and D I00 nm.

Abbreviations: RPB, high gravity rotating packed bed; SE, self-emulsification method. 

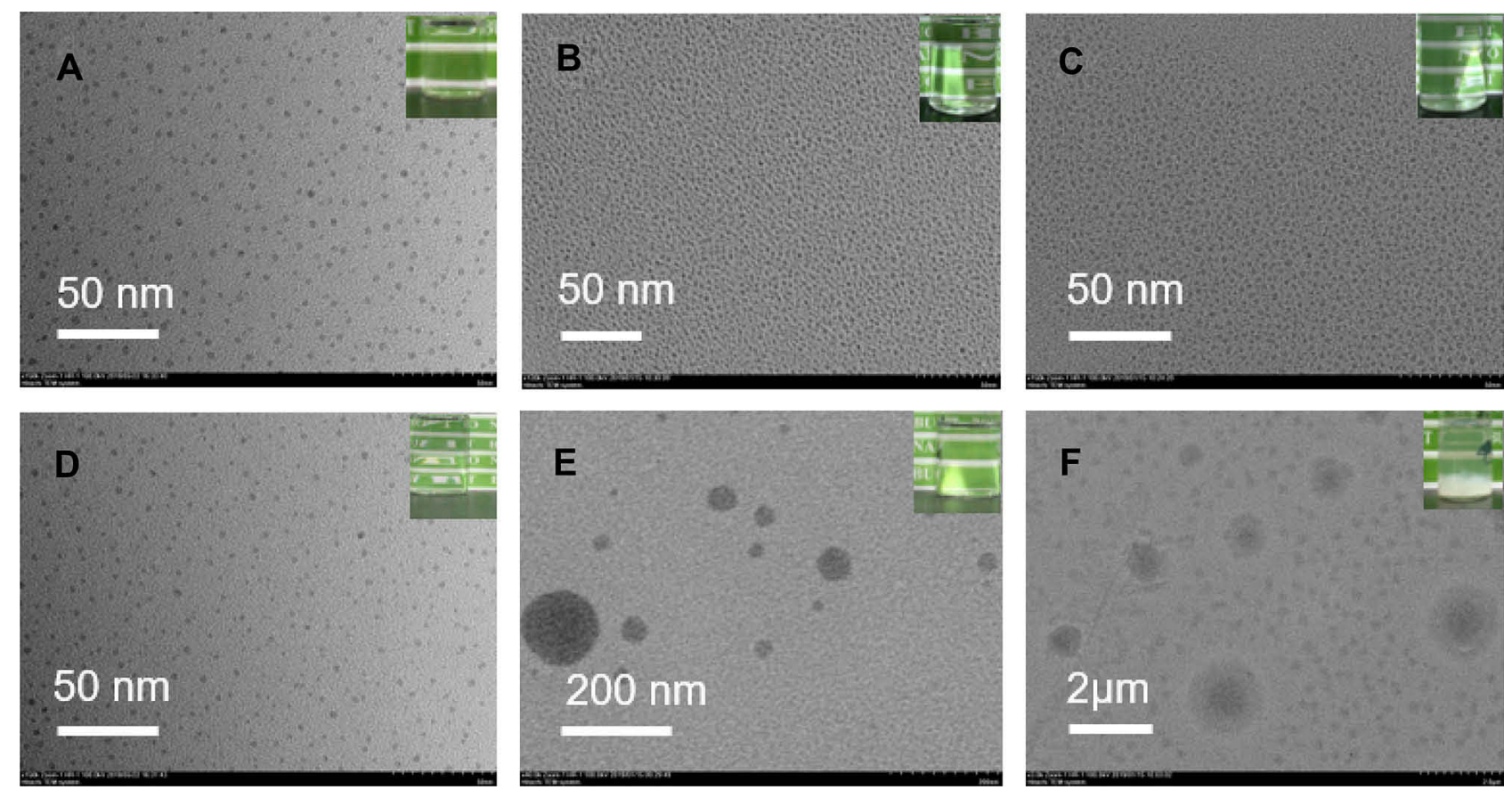

Figure 7 TEM of different nanoemulsion prepared by RPB and SE within I year.

Notes: (A) Fresh sample prepared by RPB. (B) stored at $4^{\circ} \mathrm{C}$ for I year. (C) stored at $25^{\circ} \mathrm{C}$ for I year. (D) fresh sample prepared by SE. (E) stored at $4^{\circ} \mathrm{C}$ for I year. (F) stored at $25^{\circ} \mathrm{C}$ for I year.

Abbreviations: TEM, Transmission electron microphotography; RPB, high gravity rotating packed bed; SE, self-emulsification method.

tension, thereby greatly reducing the droplet accumulation caused by the Ostwald ripening, ${ }^{29}$ therefore, the stabilization time of the RPB-prepared nanoemulsion is greatly extended. ${ }^{30}$

For both ROS and SYN nanoemulsion, the drug loading has no significant effect on droplet size as we mentioned above. In stability study, for the changes of SYN drug content, we did the study and had the same trend as well as ROS. Its drug content would be influenced by temperature and long-term storage. The RPB-prepared nanoemulsion was obliviously much better than SE samples, and the result indicated that the feasibility of RPB as a novel technique for preparing the nanoemulsion which has a prominent feature of its storage stability.

\section{Dissolution Test}

In vitro dissolution testing based on the BCS theory is the best alternative to in vivo bioequivalence studies. ${ }^{31}$ In this research, we did the dissolution test about ROS nanoemulsion compared with its raw drug and its commercial tablets but the test showed no significant differences between them because ROS is a type of BCS III drug with good water solubility.

SYN release study was performed in phosphate buffer (pH 6.8) $+0.5 \%$ Tween- 80 to simulate the endosomal condition. The drug released from nanoemulsion was extremely significant $(\mathrm{p}<0.001)$ in comparison to commercial capsules, having the same quantity of SYN (Figure 8). The SYN nanoemulsion was released at a faster rate in the phosphate buffer, after $24 \mathrm{~h}$, the drug release rate of the SYN nanoemulsion reached $95 \%$. In contrast, the commercial

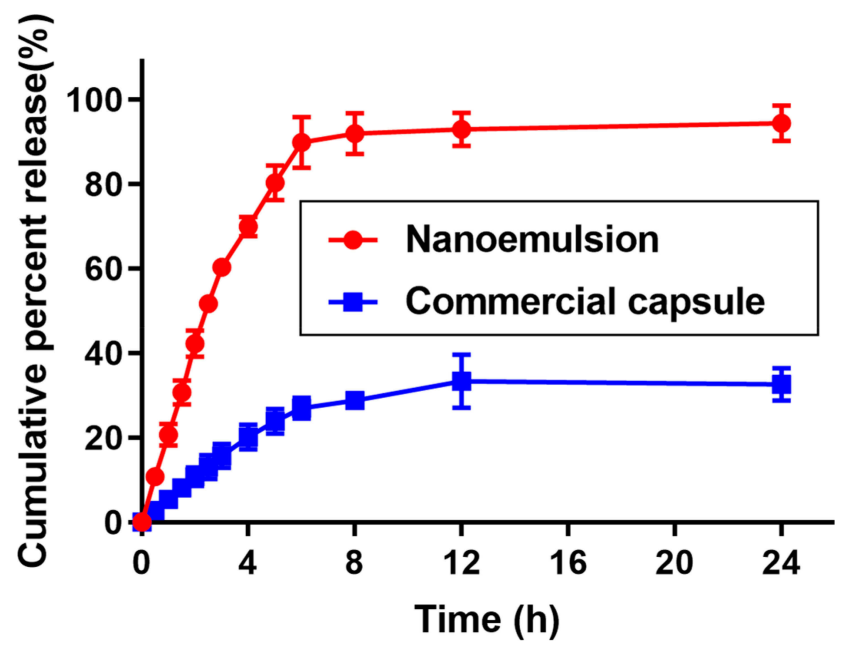

Figure 8 The dissolution test of SYN nanoemulsion and commercially capsule in $24 \mathrm{~h}$. Notes: $\mathrm{P}_{\mathrm{app}}=\frac{\mathrm{dQ}}{\mathrm{dt}} \times \frac{1}{\mathrm{AC}_{0}}$. Each data point represents the mean \pm S.D.of three determinations.

Abbreviation: SYN-NE, Silybinin nanoemulsion. 
capsules of SYN are slow to release and the release rate is significantly lower, after $24 \mathrm{~h}$, only $33 \%$ of SYN drugs are released in the phosphate buffer. The significant increase in the solubility properties of the nanoemulsion type is mainly due to the hydrophilic properties and the smaller size of the droplets.

\section{In vitro Permeability Study Across Caco-2 Cell Monolayers}

Before the in vitro permeability study, we did MTT assay to obtain drug concentration and measured the TEER value, which can ensure the integrity of Caco-2 cells monolayers. MTT assay was conducted to assess the cytotoxic effects of nanoemulsion. Nanoemulsion showed non-significant cytotoxicity (cell viability $>80 \%$ ) at the concentration between 5 and $20 \mu \mathrm{g} / \mathrm{mL}$. Thus, the concentration of $20 \mu \mathrm{g} / \mathrm{mL}$ was selected for the in vitro permeability study.

We measured Caco-2 permeation of the as-prepared drug nanoemulsion and compared with that of the commercial formulation. Figure 9 displays the permeability of BCSIII drug ROS in $2 \mathrm{~h}$. When absorptive transport from apical to basolateral (A to B), ROS raw material hardly passed, commercial tablets passed a little with $\mathrm{P}_{\text {app }}$ of $1.03 \times 10^{-5} \mathrm{~cm} / \mathrm{s}$, while the ROS nanoemulsion could smoothly pass through with $\mathrm{P}_{\mathrm{app}}$ of $3.64 \times 10^{-5} \mathrm{~cm} / \mathrm{s}(\mathrm{p}<0.001)$. When efflux from basolateral to apical ( $\mathrm{B}$ to $\mathrm{A}$ ), the cumulative transport rate of the ROS raw material was $4.48 \times 10^{-5} \mathrm{~cm} / \mathrm{s}$, higher than that of commercial tablets $4.36 \times 10^{-5} \mathrm{~cm} / \mathrm{s}$ and ROS nanoemulsion

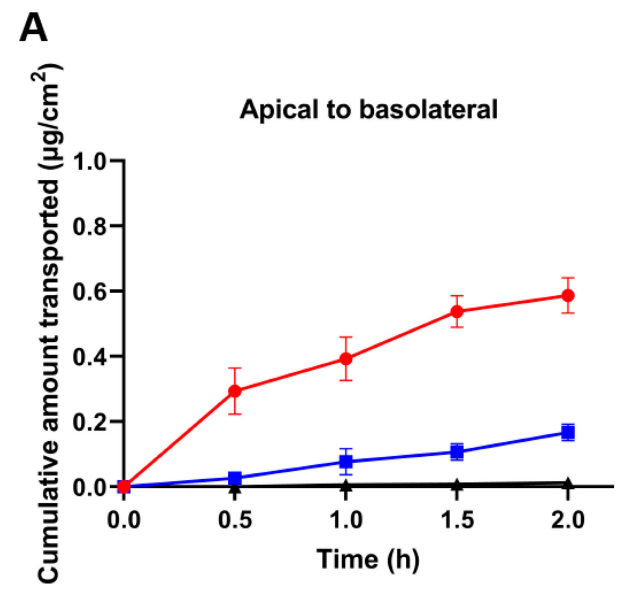

$3.41 \times 10^{-5} \mathrm{~cm} / \mathrm{s}(\mathrm{p}<0.01)$. ROS nanoemulsion was extremely significant by comparing with raw ROS and its commercial tablet. The results demonstrated that ROS nanoemulsions improved permeation over commercial tablets.

Permeability of BCSII drug SYN is shown in Figure 10. In the apical to basolateral direction (A to B), the nanoemulsion has the highest cumulative amount transported of $10.39 \times 10^{-5}$ $\mathrm{cm} / \mathrm{s}$, which is 1 -fold than that of commercial capsules $5.36 \times 10^{-5} \mathrm{~cm} / \mathrm{s}(\mathrm{p}<0.005)$; in the basolateral to apical direction (B to A), Papp of nanoemulsion was $7.56 \times 10^{-5} \mathrm{~cm} / \mathrm{s}$, quite smaller than that of capsules $12.72 \times 10^{-5} \mathrm{~cm} / \mathrm{s}(\mathrm{p}<0.01)$, which indicated nanoemulsion also has the effect of improving the permeability of BCSII drugs. Statistically, SYN nanoemulsion had a significant difference compared with its commercial capsules.

Compared with conventional oral capsules and tablets, nanoemulsion formulation not only enhanced the permeability on the Caco- 2 cell monolayers but also inhibited the efflux effect of P-glycoprotein. The main reason is due to the use of surfactants and the formation of small oil-inwater droplets. $^{32-34}$ Therefore, nanoemulsion dosage form, it is possible to effectively solubilize the drug, avoid the first-pass effect of the drug, improve cell permeability, and ultimately lead to an increase in bioavailability.

\section{Conclusions}

In summary, stable nanoemulsion for oral drug delivery was successfully prepared by high-gravity technology, which offered a platform for continuous manufacturing. Emulsifier

\section{B}

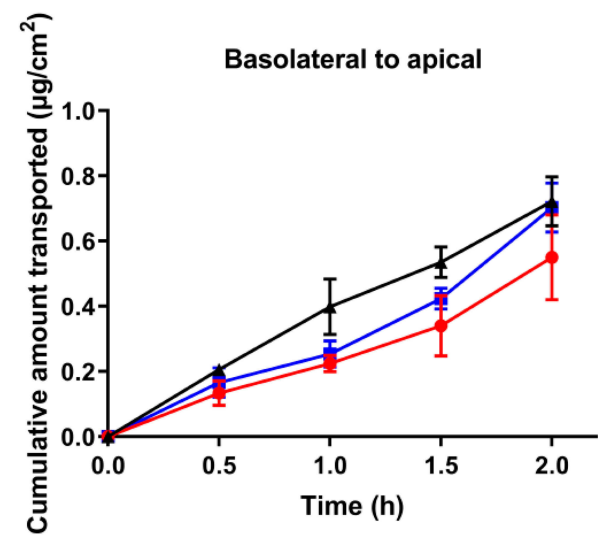

\section{$\rightarrow$ ROS-NE $\rightarrow$ Commercially tablet $₫$ Raw}

Figure 9 Cell permeability results of ROS.

Notes: (A) apical to basolateral and (B) basolateral to apical transport profiles of nanoemulsion, commercially tablet and raw ROS across the Caco- 2 cell monolayer at $37^{\circ}$ C. Each data point represents the mean \pm S.D.of three determinations.

Abbreviations: ROS, Rosuvastatin calcium. ROS-NE, Rosuvastatin calcium nanoemulsion. Raw, raw material. 
A

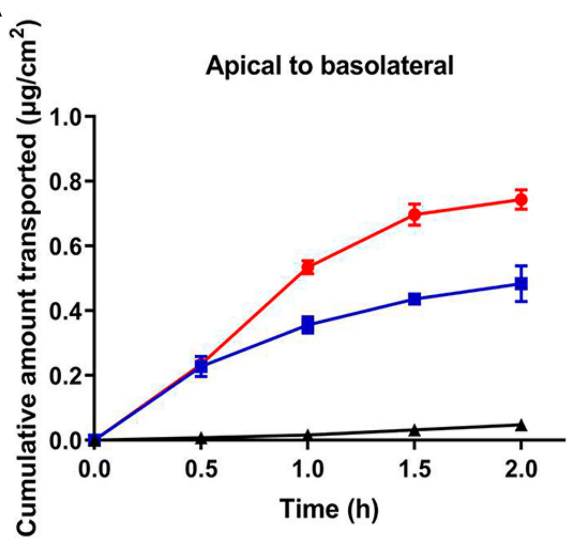

B

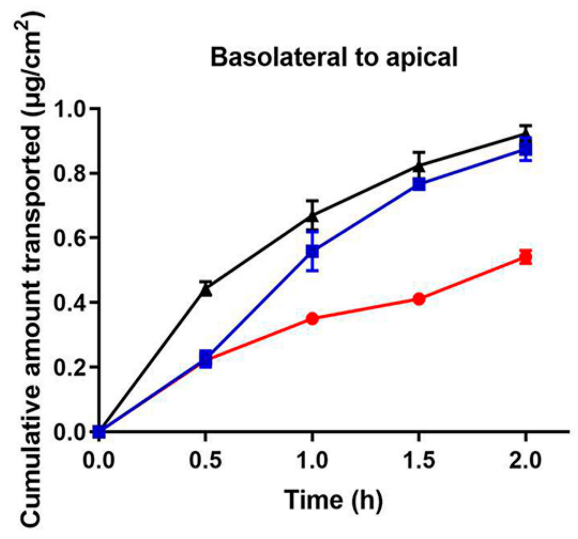

$\rightarrow$ SYN-NE $\rightarrow$ Commercial capsule $\quad \leftarrow$ Raw

Figure 10 Cell permeability results of SYN.

Notes: (A) apical to basolateral and (B) basolateral to apical transport profiles of nanoemulsion, commercially capsule and raw SYN across the Caco-2 cell monolayer at $37^{\circ} \mathrm{C}$. Each data point represents the mean \pm S.D.of three determinations.

Abbreviations: SYN, Silybinin; SYN-NE, Silybinin nanoemulsion; Raw, Raw Material.

dosage was greatly reduced by using RPB so that the biosafety of the emulsion was improved. Droplets of the nanoemulsion prepared by RPB were $14 \mathrm{~nm}$ with narrow size distribution. Meanwhile, nanoemulsion greatly enhanced the BCS class II drug SYN release rate, and the apparent permeability of the BCS class III drug ROS and BCS class II drug SYN were both enhanced. Moreover, the appearance of drug nanoemulsion kept clear and transparent without size change during 1-year storage at $25^{\circ} \mathrm{C}$ and $4^{\circ} \mathrm{C}$.

\section{Acknowledgments}

This work was supported by the National Natural Science Foundation of China (21622601).

\section{Disclosure}

The authors report no conflicts of interest in this work.

\section{References}

1. HE XW, Zhao YM, Bao Q, YE XL, LI FZ. Nanocarriers for enhancing the oral bioavailability of poorly soluble drugs: research advances. J Int Pharm Res. 2011;38(3):212-215.

2. Emami S, Siahi-Shadbad M, Adibkia K, Barzegar-Jalali M. Recent advances in improving oral drug bioavailability by cocrystals. BioImpacts: BI. 2018;8(4):305-320. doi:10.15171/bi.2018.33

3. McClements DJ, Rao J. Food-grade nanoemulsions: formulation, fabrication, properties, performance, biological fate, and potential toxicity. Crit Rev Food Sci Nutr. 2011;51(4):285-330. doi:10.1080/ 10408398.2011.559558

4. Wu CY, Benet LZ. Predicting drug disposition via application of BCS: transport/absorption/elimination interplay and development of a biopharmaceutics drug disposition classification system. Pharm Res. 2005;22(1):11-23. doi:10.1007/s11095-004-9004-4
5. Gibaldi M, Boyes RN, Feldman S. Influence of first-pass effect on availability of drugs on oral administration. J Pharm Sci. 2010;60 (9):1338-1340. doi:10.1002/jps.2600600909

6. Zhao L, Wei Y, Huang Y, He B, Zhou Y, Fu J. Nanoemulsion improves the oral bioavailability of baicalin in rats: in vitro and in vivo evaluation. Int $J$ Nanomedicine. 2013;8:3769-3779. doi:10.2147/IJN.S51578

7. Mason TG, Wilking JN, Meleson K, Chang CB, Graves SM. Nanoemulsions: formation, structure, and physical properties. $J$ Phys Condens Matter. 2006;18(41):635-666. doi:10.1088/09538984/18/41/R01

8. Rai VK, Mishra N, Yadav KS, Yadav NP. Nanoemulsion as pharmaceutical carrier for dermal and transdermal drug delivery: formulation development, stability issues, basic considerations and applications. J Control Release. 2018;270:203-225.

9. McClements DJ. Recent developments in encapsulation and release of functional food ingredients: delivery by design. Curr Opin Food Sci. 2018;23:80-84. doi:10.1016/j.cofs.2018.06.008

10. Yukuyama MN, Ghisleni DDM, Pinto TJA, Bou-Chacra NA. Nanoemulsion: process selection and application in cosmetics - a review. Int $J$ Cosmet Sci. 2016;38(1):13-24. doi:10.1111/ics. 2016.38.issue-1

11. Singh Y, Meher JG, Raval K, et al. Nanoemulsion: concepts, development and applications in drug delivery. $J$ Control Release. 2017;252:28-49. doi:10.1016/j.jconrel.2017.03.008

12. Jafari SM, Assadpoor E, He Y, Bhandari B. Re-coalescence of emulsion droplets during high-energy emulsification. Food Hydrocoll. 2008;22(7):1191-1202. doi:10.1016/j.foodhyd.2007.09. 006

13. Sutradhar KB, Amin ML. Nanotechnology in Cancer Drug Delivery and Selective Targeting. ISRN Nanotechnol. 2014;2014:1-12. doi:10.1155/2014/939378

14. Mcclements DJ. Edible nanoemulsions: fabrication, properties, and functional performance. Soft Matter. 2011;7(6):2297-2316. doi:10.1039/COSM00549E

15. Tadros T, Izquierdo R, Esquena J, Solans C. Formation and stability of nano-emulsions. Adv Colloid Interface Sci. 2004;108:303-318.

16. Solans C, Izquierdo P, Nolla J, Azemar N, Garcia-Celma MJ. Nanoemulsions. Curr Opin Colloid In. 2005;10(3):102-110. doi:10.1016/j. cocis.2005.06.004 
17. Kuang YY, Zhang ZB, Xie ML, Wang JX, Le Y, Chen JF. Large-scale preparation of amorphous cefixime nanoparticles by antisolvent precipitation in a high-gravity rotating packed bed. Ind Eng Chem Res. 2015;54(33):8157-8165. doi:10.1021/acs.iecr.5b01584

18. Zhang ZL, Le Y, Wang JX, Zhao H, Chen JF. Development of stabilized itraconazole nanodispersions by using high-gravity technique. Drug Dev Ind Pharm. 2012;38(12):1512-1520. doi:10.3109/03639045.2012.654794

19. Chen JF, Zhou MY, Shao L, et al. Feasibility of preparing nanodrugs by high-gravity reactive precipitation. Int $J$ Pharm. 2004;269 (1):267-274. doi:10.1016/j.ijpharm.2003.09.044

20. Chen JF, Wang YH, Guo F, Wang XM, Zheng C. Synthesis of nanoparticles with novel technology: high-gravity reactive precipitation. Ind Eng Chem Res. 2000;39(4):948-954. doi:10.1021/ ie990549a

21. Porter CJH, Trevaskis NL, Charman WN. Lipids and lipid-based formulations: optimizing the oral delivery of lipophilic drugs. Nat Rev Drug Discov. 2007;6(3):231-248. doi:10.1038/nrd2197

22. Lin JH, Yamazaki M. Role of P-glycoprotein in pharmacokinetics: clinical implications. Clin Pharmacokinet. 2003;42(1):59-98. doi:10.2165/00003088-200342010-00003

23. Li PH, Lu WC. Effects of storage conditions on the physical stability of D-limonene nanoemulsion. Food Hydrocoll. 2016;53 (FEB):218-224. doi:10.1016/j.foodhyd.2015.01.031

24. Yu H, Huang Q. Investigation of the absorption mechanism of solubilized curcumin using Caco-2 cell monolayers. J Agric Food Chem. 2011;59(17):9120-9126. doi:10.1021/jf201451m

25. Nassar T, Rom A, Nyska A, Benita S. A novel nanocapsule delivery system to overcome intestinal degradation and drug transport limited absorption of p-glycoprotein substrate drugs. Pharm Res. 2008;25 (9):2019-2029. doi:10.1007/s11095-008-9585-4
26. Bhushani JA, Karthik P, Anandharamakrishnan C. Nanoemulsion based delivery system for improved bioaccessibility and Caco-2 cell monolayer permeability of green tea catechins. Food Hydrocoll. 2016;56:372-382. doi:10.1016/j.foodhyd.2015.12.035

27. Li YJ, Hu XB, Lu XL, et al. Nanoemulsion-based delivery system for enhanced oral bioavailability and Caco-2 cell monolayers permeability of berberine hydrochloride. Drug Deliv. 2017;24(1):1868-1873. doi:10.1080/10717544.2017.1410257

28. Liu XZ, Jin JS, Chen YL, et al. Controllable polymerization of n-butyl cyanoacrylate using a high-gravity rotating packed bed. Chem Eng J. 2020;379:122400. doi:10.1016/j.cej.2019.122400

29. Yao JH, Elder KR, Guo H, Grant M. Theory and simulation of Ostwald ripening. Phys Rev B Condens Matter. 1993;47 (21):14110-14125. doi:10.1103/PhysRevB.47.14110

30. Shah P, Bhalodia D, Shelat P. Nanoemulsion: a pharmaceutical review. Sys Rev Pharm. 2010;1(1):24-31. doi:10.4103/09758453.59509

31. Polli JE, Abrahamsson BSI, Yu LX, et al. Summary workshop report: bioequivalence, biopharmaceutics classification system, and beyond. AAPS J. 2008;10(2):373-379. doi:10.1208/s12248-008-9040-9

32. Yin YM, Cui FD, Mu CF, et al. Docetaxel microemulsion for enhanced oral bioavailability: preparation and in vitro and in vivo evaluation. J Control Release. 2009;140(2):86-94. doi:10.1016/j. jconrel.2009.08.015

33. Buggins TR, Dickinson PA, Taylor G. The effects of pharmaceutical excipients on drug disposition. Adv Drug Del Rev. 2007;59 (15):1482-1503. doi:10.1016/j.addr.2007.08.017

34. Yamagata T, Kusuhara H, Morishita M, Takayama K, Benameur H, Sugiyama Y. Effect of excipients on breast cancer resistance protein substrate uptake activity. J Control Release. 2007;124(1):1-5. doi:10.1016/j.jconrel.2007.08.021
International Journal of Nanomedicine

\section{Publish your work in this journal}

The International Journal of Nanomedicine is an international, peerreviewed journal focusing on the application of nanotechnology in diagnostics, therapeutics, and drug delivery systems throughout the biomedical field. This journal is indexed on PubMed Central, MedLine, CAS, SciSearch ${ }^{\mathbb{}}$, Current Contents ${ }^{\mathbb{R}} /$ Clinical Medicine,

\section{Dovepress}

Journal Citation Reports/Science Edition, EMBase, Scopus and the Elsevier Bibliographic databases. The manuscript management system is completely online and includes a very quick and fair peer-review system, which is all easy to use. Visit http://www.dovepress.com/ testimonials.php to read real quotes from published authors. 